\title{
Inhibition of EGFR nuclear shuttling decreases irradiation resistance in HeLa cells
}

\author{
Hong Wei, Zijie Zhu, Longtao Lu
}

Central Laboratory of Weifang Hospital of Traditional Chinese Medicine, Weifang, Shangdong Province, China

\begin{abstract}
Introduction. Cervical cancer is a leading cause of mortality in women worldwide. The resistance to irradiation at the advanced stage is the main reason for the poor prognosis and high mortality. This work aims to elucidate the molecular mechanism underlying the radio-resistance.

Material and methods. In this study, we determined the pEGFR-T654 and pDNA-PK-T2609 expression level changes in irradiated HeLa cells treated with T654 peptide, a nuclear localization signal (NLS) inhibitor, to inhibit EGFR nuclear transport. Cell viability, cell cycle and migratory capacity were analyzed. Xenograft animal model was used to evaluate the effect of EGFR nuclear transport inhibition on the tumor growth in vivo.

Results. The enhanced translocation of nuclear EGFR in the irradiated HeLa cells correlated with the increasing level of pEGFR-T654 and pDNA-PK-T2609. Inhibition of EGFR nuclear translocation by NLS peptide inhibitor attenuated DNA damage repair in the irradiated HeLa cells, decreased cell viability and promoted cell death through arrest at G0 phase. NLS peptide inhibitor impaired the migratory capacity of irradiated HeLa cells, and negatively affected tumorigenesis in xenograft mice.

Conclusions. This work puts forward a potential molecular mechanism of the irradiation resistance in cervical cancer cells, providing a promising direction towards an efficient therapy of cervical cancer. (Folia Histochemica et Cytobiologica 2017, Vol. 55, No. 2, 43-51)
\end{abstract}

Key words: EGFR; HeLa; nuclear translocation; pEGFR-T654; pDNA-PK-T2609; NLS peptide inhibitor; cell migration; xenograft

\section{Introduction}

Cervical cancer is the leading cause of cancer-associated mortality in women [1]. Although the great progress has been made in the diagnosis and treatment of cervical cancer, the five year survival rate is still low due to the tumor metastasis and drug resistance [2]. One of the most commonly used treatments for cervical cancer is radiotherapy. However, radiation resistance causes inefficiency of irradiation therapy,

Correspondence address: $\mathrm{H}$. Wei

Central Laboratory of Weifang Hospital of Traditional

Chinese Medicine

Weifang, Shangdong Province, China

No. 1055 Weizhou Road, Kuiwen District

tel.: 86-0536-8060011

e-mail: hongwei750@yahoo.com especially in the patients at advanced stage. Therefore, resistance to radiation is considered as a poor indicator to prognosis [3].

In order to clarify the underlying mechanism of radiation resistance, several studies indicated the critical genes involved in the process of radio-resistance [4-8]. Most recently, it has been reported that mutation in epidermal growth factor receptor (EGFR) correlated with the results of cervical cancer treatment [9]. EGFR is one of four members of the ErbB family of receptor tyrosine kinases. The nuclear translocation of EFGR was reported to be involved in the resistance to cancer therapy $[12,13]$. Nuclear localization signal (NLS) of EGFR that plays a critical role in the nuclear translocation [15] has been considered as a key factor to the resistance to cancer therapy [13]. However, the function of the nuclear translocation of EGFR in cervical cancer has not been well studied. 
In the current study, we elucidated the role of EGFR nuclear translocation in the radiosensitivity of cervical cancer cells. We found that inhibition of nuclear translocation could decrease the survival rate and attenuate the migration capacity of cervical cancer cells after irradiation. It also reduced tumorigenesis in xenograft nude mice models in vivo.

\section{Material and methods}

Cell culture and irradiation exposure. HeLa cell line was purchased from ATCC (Manassas, VA, USA). The cultures were maintained in DMEM supplemented with $10 \%$ fetal bovine serum (Life Technologies, Carlsbad, CA, USA). The cells were grown at $37^{\circ} \mathrm{C}$ in a humidified incubator in an atmosphere of $5 \% \mathrm{CO}_{2}$ and passaged every 3 days. Cells were exposed to a single dose of 4 Gy of X-rays using the $200-\mathrm{kV}$ photons linear accelerator (Gulmay RS 225, Byfleet, UK) at a dose rate of $450 \mathrm{cGy} / \mathrm{min}$ for $40 \mathrm{~min}$ at $37^{\circ} \mathrm{C}$.

Construction of EGFP-C1-EGFR plasmid and establishment of EGFR-overexpressed cell line. Human full length EGFR cDNA was ligated into EGFR-C1 plasmid. The insertion was confirmed by sequencing. The EGFR plasmid was transfected into Hela cells by Lipofectamine 2000 (Life Technologies). The cells stably expressing EGFR was selected by neomycin. The overexpression of EGFR in selected cell lines was confirmed by GFP fluorescence and immunoblotting against EGFR.

Western blot. $2 \mu \mathrm{g}$ of cell lysates were loaded on each lane of $10 \%$ polyacrylamide gel, and then blotted onto a polyvinylidene difluoride (PVDF) membrane. After blocking with a PBST (phosphate-buffered saline containing 0.3\% Trition-X100) containing 5\% nonfat dry milk, the membrane was incubated with antibodies against EGFR, phosphorylated EGFR at T654 (p-EGFR-T654), and phosphorylated DNA-PK at T2609 (p-DNA-PK-T2609) (Cell Signaling Technologies, MA, USA). Peroxidase-linked anti rabbit IgG (Life Technologies) were used as secondary antibodies. These proteins were visualized by using an ECL Western blotting detection kit (Amersham Biosciences, Little Chalfont, UK).

Subcellular fractionation. Cytoplasmic and nuclear extracts were prepared according to the instructions of the NE-PER ${ }^{\circledR}$ nuclear and cytoplasmic extraction kit (Pierce, Rockford, IL, USA).

T654-peptide and control peptide. T654-peptide (EGFR NLS peptide inhibitor RKRT(PO3H2)LRRLK) and control peptide (KKALRRQEAVNAL) were synthesized by GL Biochem (Shanghai, China). Cells were treated with $5 \mu \mathrm{M}$ peptide for 12 hours. The function of inhibitor and control peptides was confirmed in the previous report [16].
Cell viability assessment. MTT assay was used to detect effect of T654-peptide on cell viability of irradiated HeLa. Briefly, MTT also known as 3-(4,5-dimethylthiazol-2-yl)-2,5-diphenyltetrazolium bromide (Sangon Biotech, Shanghai, China) was incubated with cells for 4 hrs to produce formazan salts. When formazan was completely dissolved by SDS- $\mathrm{HCl}$, the absorbance at $570 \mathrm{~nm}$ was measured with a Universal Microplate Reader (Bio-Tek Instruments, Winooski, VT, USA), and the ratio of the optical density (OD) of sample group/OD of blank control was calculated.

TUNEL assay. Terminal deoxynucleotidyl transferase-mediated dUTP nick end labeling (TUNEL) assay was performed by using an in situ cell death detection kit (Santa Cruz Biotechnology, Dallas, TX, USA). Briefly, thyroid sections were fixed with $4 \%$ paraformaldehyde and permeabilized with $0.2 \%$ Triton $\mathrm{X}-100$. Fragmented DNA was labeled with fluorescein-12-dUTP at $37^{\circ} \mathrm{C}$ for 1 hour. TUNEL-positive nuclei were detected with Eclipse E1000 fluorescent microscope (Nikon, Tokyo, Japan).

Immunohistochemistry. The sections of xenograft tumors were washed in PBS, blocked for 60 min in $0.3 \%$ Triton $\mathrm{X}-100$ in PBS with $5 \%$ bovine serum albumin, and then incubated at $4^{\circ} \mathrm{C}$ with anti-Ki67 antibodies (Abcam, Cambridge, UK) overnight. FITC-conjugated secondary antibody was used to visualize the positive cells. Ratio of Ki67-positive cell was measured by Nikon Eclipse E1000 fluorescent microscope.

RNA extraction and qRT-PCR. Total RNA extraction was performed using TRIzol reagent (Life Technologies) according to the manufacturer's instruction. Two micrograms of total RNA extracted from the cells was subjected to reverse transcription (RT). Synthesis of cDNA was performed by using one-step RT-PCR kit from Takara (Dalian, China). SYBR Green (Toyobo, Osaka, Japan) RT-PCR amplification and real time fluorescence detection were performed using ABI 7300 real-time PCR thermal cycle instrument (ABI, Foster City, CA, USA), according to the supplied protocol. Relative gene expression was calculated by the $\Delta \Delta \mathrm{Ct}$ method. Primers used for Ki67 are as follows: 5'-ATAAACACCCCAACACACACAA-3' and 5'-GCCACTTCTTCATCCAGTTAC-3'. The relative expression levels were normalized to the expression of endogenous GAPDH (5'-GGTATCGTGGAAGGACTCATGAC-3' and 5'ATGCCAGTGAGCTTCCCGT TCAGC-3').

Xenograft tumor models. BALB/c nude mice were purchased from Shanghai Lab Animal Research Center (Shanghai, China). All the animal handling was conformed to the protocols approved by the Institutional Animal Care and Use Committee of Weifang Hospital of Traditional Chinese Medicine. According to previous study [17], inoculation 


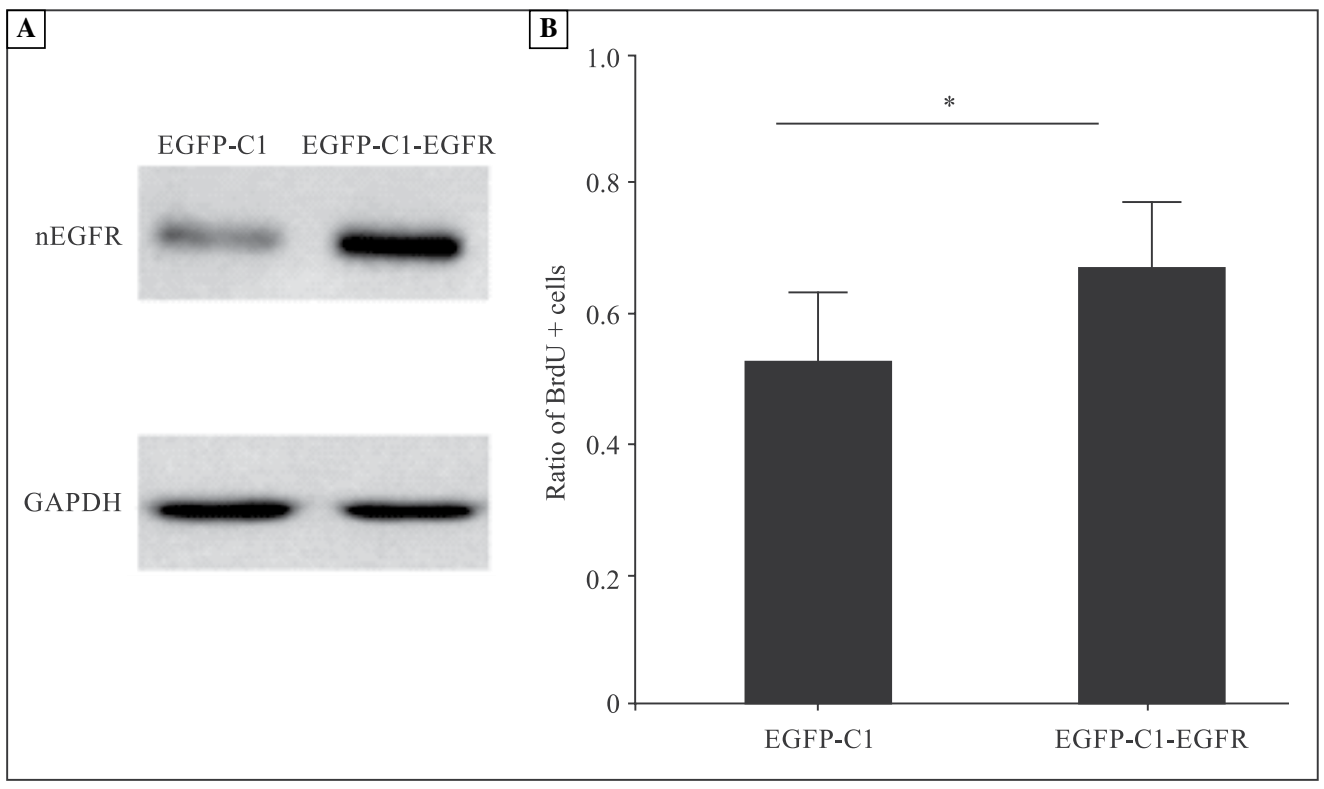

Figure 1. The effects of the epithelial growth factor receptor (EGFR) overexpression in HeLa cells on the level of nuclear EGFR (nEGFR) (A) and cell proliferation (B). Overexpression of EGFR enhanced proliferation when HeLa cells were exposed to irradiation $(n=801$ cells from three independent preparation). The way of overexpressing EGFR was described in Material and methods.

area of the mice was cleaned and sterilized with ethanol and iodine solutions. $1 \times 10^{5}$ irradiated HeLa cells in PBS treated by a control peptide (a peptide with the scramble amino acid sequence similar with T654-peptide but without the biological function) or T654-peptide were injected subcutaneously into the right flank of 6 weeks-old BALB/c nude mice. Each group contained six mice, and xenograft experiments were conducted in triplicate. Obvious tumor was observed 4 weeks after cell injection. The implanted mice were observed daily until 60 days. Tumor volume (V) was calculated using the following equation: Tumor volume $\left(\mathrm{mm}^{3}\right)=0.5 \times$ length $\times$ width $^{2}$.

Analysis of cell cycle phase by flow cytometry. Twelve hours following the treatment with control peptide or T654 peptide, the HeLa cells were resuspended in PBS twice before fixation by adding dropwise into to $95 \%$ precooled ethanol. Prior to analysis, the cells were warmed, centrifuged at $450 \mathrm{~g}$ for $5 \mathrm{~min}$ and resuspended twice in PBS, then stained with propidium iodide containing RNase A at $50 \mu \mathrm{g} / \mathrm{mL}$ (Sangon, Shanghai, China) at room temperature in the dark for $30 \mathrm{~min}$. The DNA content was analyzed by flow cytometry using the CellQuest program (Becton-Dickinson, Franklin Lakes, NJ, USA).

Scratch wound assay. Cells were seeded on $60 \mathrm{~cm}^{2}$ tissue-culture plastic dishes at $80 \%$ cell confluence. A scratch wound was performed using a sterile $200 \mu \mathrm{L}$ pipette tip. Phase-contrast images were taken at different time points.
The distance between the scratch line and the number of cells which crossed the scratch line were measured.

Statistical analysis. For statistical analysis, all data were obtained from three independent experiments. Data were presented as mean \pm SEM. The band density in the Western blot analysis was measured by using Image $\mathbf{J}$ software (developed at the National Institutes of Health, Bethesda, MD, USA). Unpaired Student's t-test was used to determine significant differences. A p value less than 0.05 was considered as significantly different.

\section{Results}

\section{Radiation induced the phosphorylation of EGFR at Thr654}

Firstly, we elucidated whether the EGFR nuclear translocation could affect the proliferation of cervical cancer cells. The overexpression of EGFR led to the increased level of nuclear EGFR in HeLa cells (Fig. 1A). The nuclear accumulation of EFGR in HeLa cells was associated with enhanced proliferation after exposure to irradiation (Fig. 1B, p < 0.05). Then, we determined whether the radiation could affect the expression of EGFR in the nucleus (nEGFR) of HeLa cells. The nEGFR level was significantly increased after irradiation, indicating the initiation of nuclear transport of EGFR evoked by irradiation (Fig. 2A, B). Meanwhile, the phosphorylation of EGFR at Thr654 


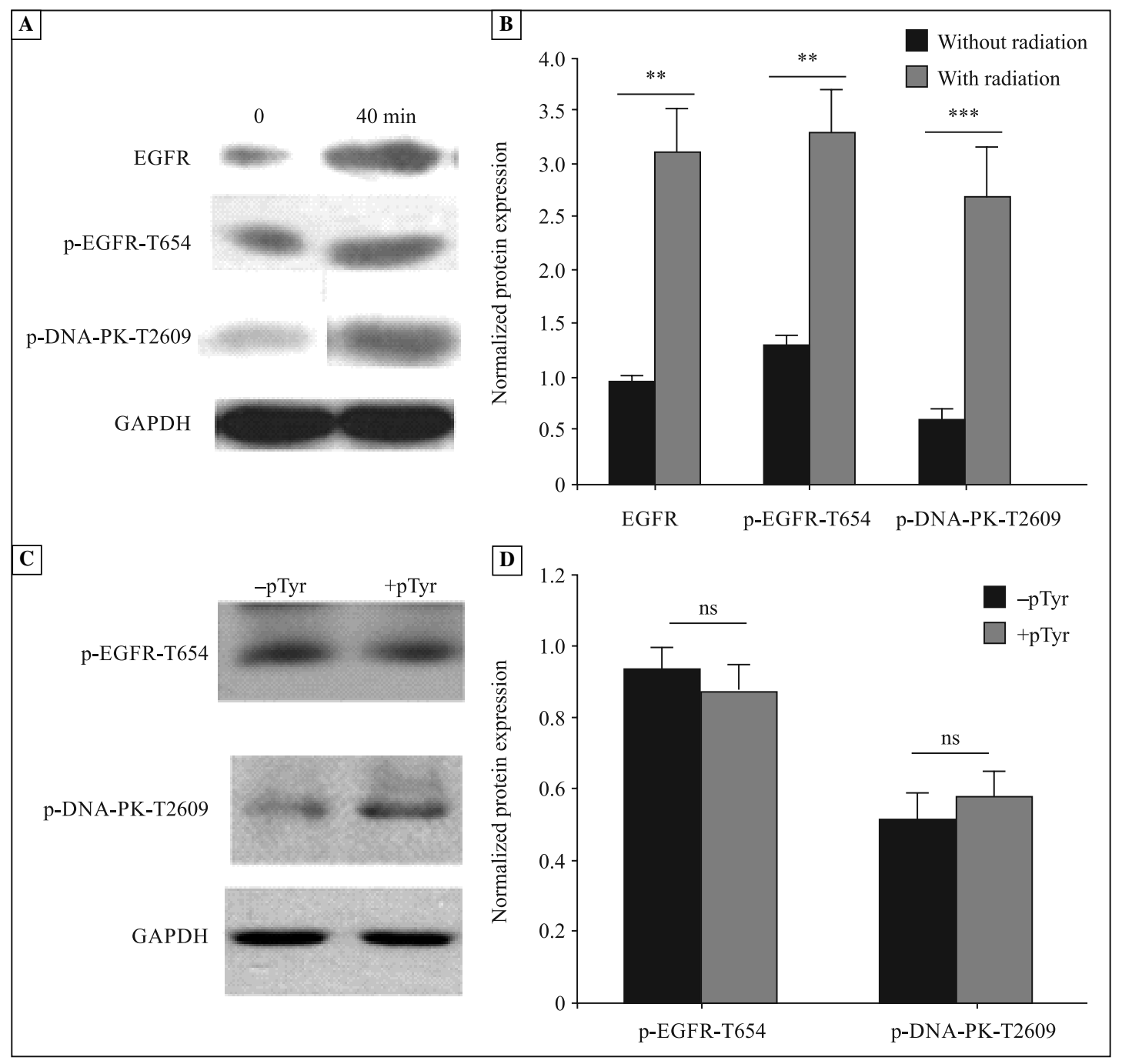

Figure 2. The effects of irradiation on the characteristics of control and modified HeLa cells. A. Western blotting analysis indicated that EGFR expression in nuclei of HeLa cells was increased when exposed to irradiation for 40 min. The phosphorylation of EGFR at Thr654 (p-EGFR-T654) and of DNA-dependent protein kinase at Thr2609 (p-DNA-PK-T2609) showed the similar pattern; B. The increase in EGFR, p-EGFR-T654, and p-DNA-PK-T2609 level of irradiated HeLa cells was significantly different from the untreated control; unpaired Student's t test; ** $\mathrm{p}<0.01$ and ***p $<0.001$; C, D. Treatment of ortho-phospho-L-tyrosine (p-Tyr) treatment did not alter the level of p-EGFR and p-DNA-PK in HeLa cells; ns — not significant.

(pEGFR-T654) was drastically increased at $40 \mathrm{~min}$ after exposure to radiation (Fig. 2A, B). Enhanced pEGFR-T654 was accompanied by the increased phosphorylation level of DNA-PK at T2609 (p-DNA-PK-T2609), which showed that elevated pEGFR-T654 level after radiation correlated with DNA repair (Fig. 2A, B). We did not observe any alterations in pEGFR-T654 and p-DNA-PK-T2609 levels in the presence of $\mathrm{p}$-Tyr, indicating the direct correlation between pEGFR-T654 and p-DNA-PK-T2609. Collectively, these data demonstrated that exposure of the HeLa cells to irradiation led to the increased phosphorylation level of EGFR at Thr654, which subsequently induced increased p-DNA-PK-T2609 level.

\section{Inhibitory effect of T654-peptide on the phosphorylation of DNA-PK}

Next, we used T654-peptide to inhibit the EGFR transport in HeLa cells. We found that T654-peptide could prevent the nuclear translocation of EGFR induced by radiation. No significantly enhanced expression of nuclear EGFR was observed in radiation-exposed HeLa cells when treated with T654-peptide (Fig. 3). The phosphorylation of EGFR was also inhibited after 


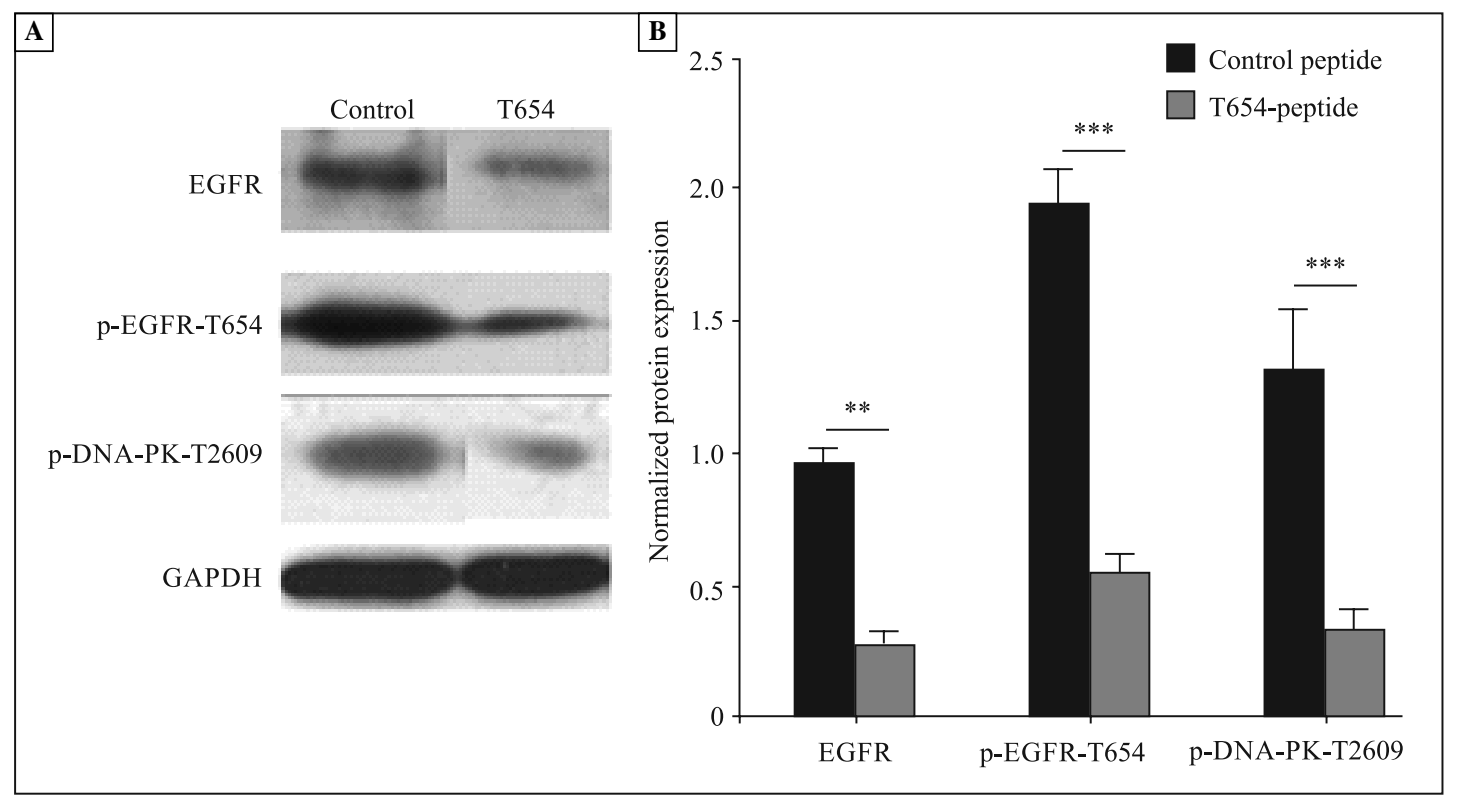

Figure 3A. Inhibition of EGFR nuclear translocation by T654-peptide treatment inhibited the increase in epithelial growth factor receptor (EGFR), phosphorylation of EGFR (p-EGFR), and phosphorylation of DNA-dependent protein kinase (p-DNA-PK) levels induced by the irradiation of HeLa cells; B. Statistical analysis showed that T654-peptide treatment significantly decreased irradiation-evoked increase in EGFR, p-EGFR, and p-DNA-PK levels compared to the cells treated with control peptide. Unpaired Student's t test; **p $<0.01$; $^{* *} \mathrm{p}<0.001$.

treatment with T654-peptide (Fig. 3). Furthermore, we did not found increased phosphorylation level of DNA-PK at T2609 in the presence of T654-peptide. This suggested that nuclear translocation of EGFR was important to the DNA repair in HeLa cells after exposure to irradiation.

\section{4-peptide enhanced HeLa cell death after radiation}

To further validate the effect of T654-peptide on the irradiated HeLa cells, we measured cell proliferation and death. The MTT assay showed that the cell viability was significantly lower after irradiation in the presence of T654-peptide, compared to the cells treated with control peptide (Fig. 4A). Cell death was also affected by the T654-peptide. The results of TUNEL assay showed that more dead cells were observed in T654-peptide-treated group (Fig. 4C) than in control peptide-treated cells (Fig. 4B). The percentage of TUNEL-positive cells drastically increased by $106 \pm$ $\pm 30 \%$ in HeLa cells treated with T654-peptide compared to the control peptide-treated cells (Fig. 4D). Furthermore, to investigate the specific effect of T654-peptide on HeLa cells, we treated EGF-nonresponsive rat cell N-3 with T654-peptide and found that $\mathrm{T} 654$-peptide did not alter $\mathrm{N}-3$ cell proliferation (Fig. 4E). Also, we did not observe any significant change in the level of p-DNA-PK T2609 (Fig. 4F), demonstrating that T654-peptide treatment did not influence DNA repair. These data demonstrated that inhibition of EGFR nuclear translocation promoted the death of HeLa cell after irradiation.

\section{T654-peptide led to the G0 arrest in irradiated HeLa cells}

Further, we determined by flow cytometry whether the T654-peptide treatment could affect the cell cycle of irradiated HeLa cells. Compared to the irradiated HeLa cells treated with control peptide, the ratio of cells at G0/G1 phase significantly increased by $123 \pm$ $24 \%$ in cells treated with T654-peptide (Fig. 5). By contrast, the ratio of cells at $\mathrm{G} 2 / \mathrm{M}$ phases was drastically decreased by $27 \pm 4 \%$ in T654-peptide-treated irradiated cells. We did not observe any significant alteration in the ratio of cells at the $\mathrm{S}$ phase (Fig. 5). This finding indicated that inhibition of EGFR nuclear translocation could prevent their radiated cells from entering the cell cycle, and then affect cell viability and induce cell death.

\section{T654-peptide inhibited the migratory ability of irradiated HeLa cells}

Next, we determined whether T654-peptide treatment could affect the migratory ability of HeLa cells after irradiation. The scratch wound assay showed that the scratch distance in T654-peptide treated HeLa cells increased by $98 \pm 16 \%$ compared to the control pep- 


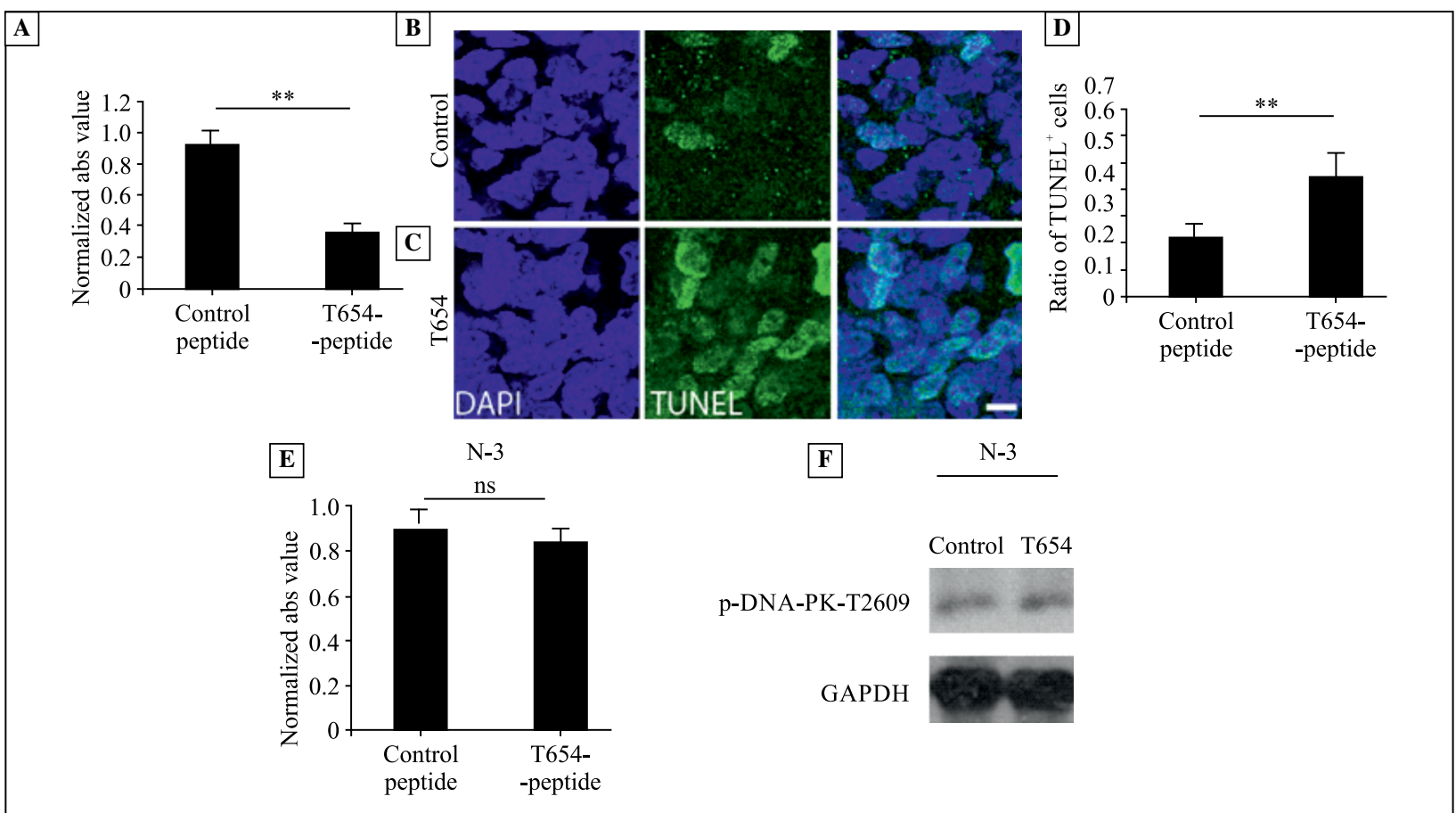

Figure 4. MTT assay showed that the viability of irradiated HeLa cells was negatively affected when treated with T654-peptide (A). More TUNEL ${ }^{+}$cells were observed in T654-peptide-treated group (C) compared to the control peptide-treated cells (B). The difference in TUNEL ${ }^{+}$cells between HeLa cells treated with T654 ( $\mathrm{n}=774$ cells from three independent preparation) or control peptide $(n=695$ cells from three independent preparation) was statistically significant (D). T654-peptide treatment did not affect the proliferation of N-3 cells $(\mathbf{E})$ or the phosphorylation level of DNA-dependent protein kinase (p-DNA-PK) (F). Unpaired Student's t test; ns - not significant; ${ }^{* *} \mathrm{p}<0.01$. Scale bar represents $20 \mu \mathrm{m}$.

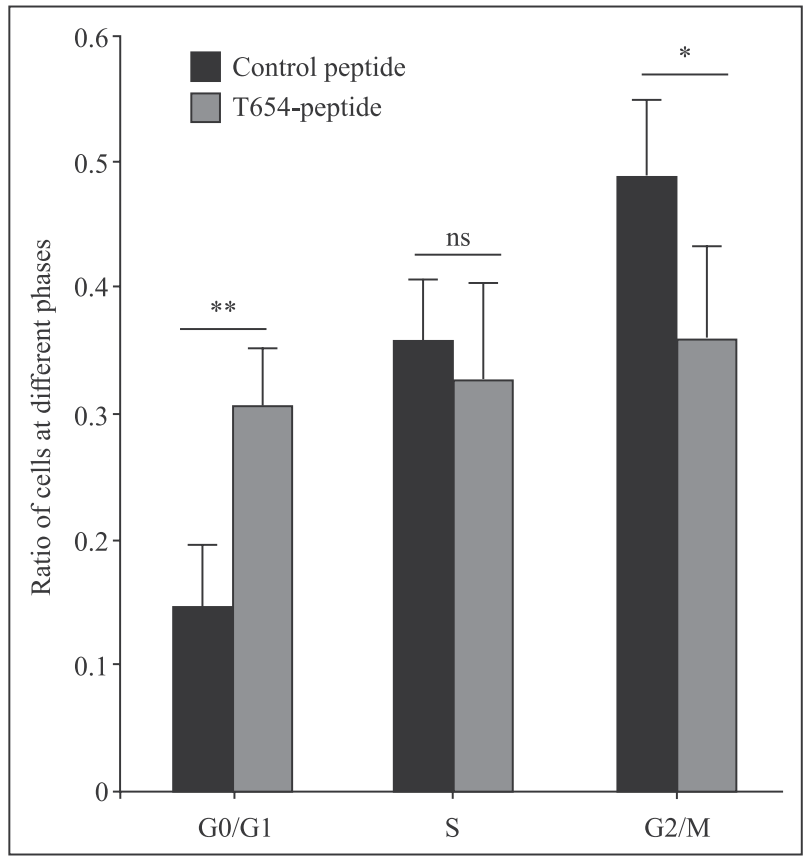

Figure 5. Cell cycle analysis showed the ratio of the HeLa cells at G0/G1 stage was increased in the presence of T654-peptide in contrast to the cells treated with control peptide, while the ratio of cells at $\mathrm{G} 2 / \mathrm{M}$ stage was decreased. Unpaired Student's t test; ns - not significant; ${ }^{*} \mathrm{p}<0.05 ;{ }^{* *} \mathrm{p}<0.01$. tide-treated cells (Fig. 6A-C). We also found that the number of cells that cross the scratch line decreased by $54 \pm 12 \%$ with the treatment with T654-peptide (Fig. 6D). These data showed that inhibition of EGFR nuclear translocation could negatively regulate migratory capacity in irradiated HeLa cells.

\section{Tumorigenesis of T654-peptide-treated HeLa cells in vivo}

Finally, we investigated the effect of T654-peptide on tumorigenesis of irradiated HeLa cells in vivo. Xenograft experiment showed that incubation of T654-peptide with the irradiated HeLa cells reduced the capacity of tumorigenesis in BALB/c nude mice (Fig. 7A). Compared to the control peptide-treated cells, the size was significantly reduced by $55 \pm$ $11 \%, 51 \pm 10 \%$, and $49 \pm 7 \%$ in tumors formed by T654-peptide-treated cells at the $30^{\text {th }}, 40^{\text {th }}$, and $50^{\text {th }}$ day after cell graft, respectively (Fig. 7B). Besides, the mRNA level of Ki67 in tumor formed by cells treated with T654-peptide was lower by $52 \pm 7 \%$ than in tumor formed by cells treated with control peptide (Fig. 7C). Immunostaining provided similar result since we found that the ratio of $\mathrm{Ki} 67^{+}$cells was lower by $48 \pm 6 \%$ in tumor formed by cells treated 


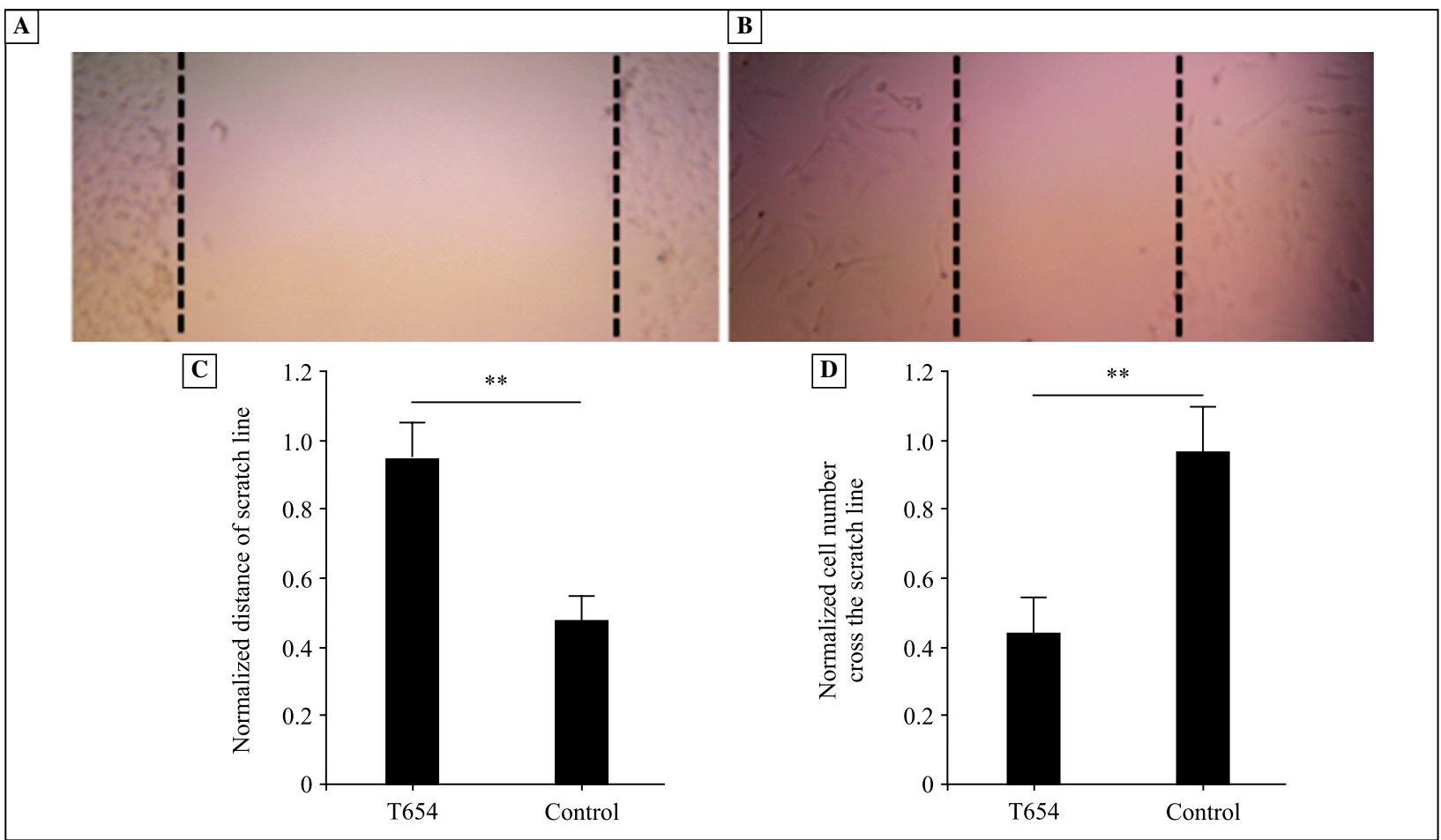

Figure 6. Images show the difference in the migration between T654-peptide-treated HeLa cells (A) and cells treated with control peptide (B). Statistical analysis showed that the distance between scratch line was significantly lower in irradiated HeLa cells treated with T654-peptide (C). The number of migratory cells crossing the scratch line was decreased in the presence of cells treated with T654-peptide compared to the control peptide-treated cells (D). Unpaired Student's t test; **p < 0.01.

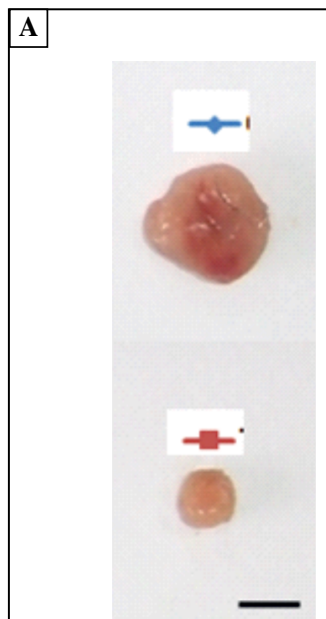

C

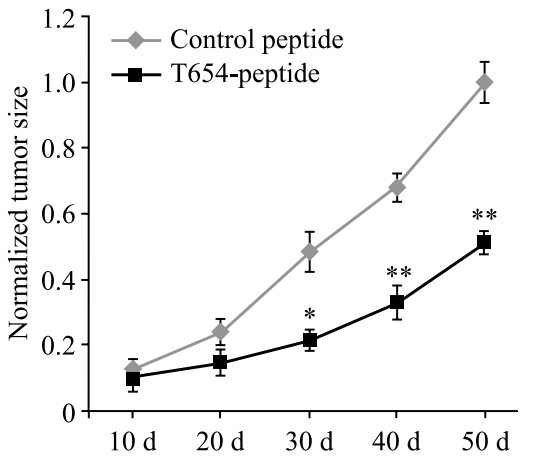

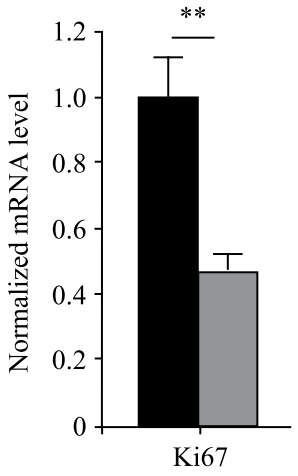

Control peptide
D

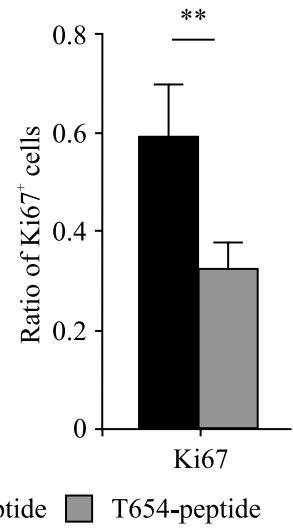

Figure 7. T654-peptide treatment of HeLa cells decreased tumor growth in vivo. A. Different size of tumor formed by control peptide treated cells (upper image) and T654-peptide treated cells (lower image); B. Significant decrease of tumor size after transplantation of cells treated with T654-peptide; C. The mRNA level of Ki67 was decreased in tumor formed by T654-peptide-treated cells; D. The ratio of Ki67 ${ }^{+}$cells was decreased in tumor formed by T654-peptide-treated cells after irradiation compared to the tumor formed by irradiated cells treated with control peptide. Unpaired Student's t test; ${ }^{* *} \mathrm{p}<0.01$.

with T654-peptide (Fig. 7D). This in vivo study further indicated that the inhibition of EGFR nuclear translocation negatively regulated the HeLa cell growth and proliferation after irradiation.

\section{Discussion}

In this study, we elucidated the role of EGFR nuclear shuttling in radiation resistance of HeLa cells. Our 
findings showed that inhibition of EGFR nuclear translocation has weakened the resistance to irradiation, reduced cell viability in vitro and attenuated the tumorigenesis in vivo. This is an important piece of evidence to support the potential clinical application of EGFR NLS peptide inhibitor in the treatment of cervical cancer.

It is well known that EGFR phosphorylation was a critical step in the activation of signaling pathway. It has been reported that several factors determined this process, including c-Src, Lyn and Yes $[18,19]$. Those factors can phosphorylate the different tyrosine sites, including Tyr 845, Tyr 992, Tyr1101 and Tyr 1173 [20, 21]. However, no direct evidence showed that these tyrosine sites were involved in the cell survival and radiation resistance. Apart from the above listed studies, the other important site for EGFR phosphorylation was threonine. Different from the function of tyrosine residues in EGFR, threonine at 654 was shown to be critical in nuclear transport and DNA damage repair in irradiated human cancer cells $[16,22]$. Our results are consistent with data obtained in these studies. However, we still did not know which factor controls the phosphorylation at T654. Other reports showed that this process resulted from the activation of PKC $\varepsilon$ and modulation of other kinase activity [23]. The underlying mechanism of T654 phosphorylation in irradiated cervical cancer cells need to be further investigated.

The other interesting finding in this work is that inhibition in EGFR nuclear transport led to the decrease in p-DNA-PK-T2609 level, showing that the ability of DNA repair in irradiated HeLa cells was to some extent impaired. Phosphorylation of DNA-PK is responsible for the activation of DNA-PK [24]. Thus, this process is critical to repair the double strand DNA breaks. Due to the lower expression level of p-DNA-PK, the survival rate of irradiated cancer cells should decrease and the ability to form the tumor should be impaired. This is exactly what we observed in our study. More work could be focused on which critical signaling pathway(s) regulated cell death in tumor cells exposed to irradiation besides the EGFR signaling elucidated by Dittmann et al. [22,25]. The potential related signaling include beta-catenin/TCF4 [26], vascular endothelial growth factor (VEGF) signaling [27], and downstreaming p44/42 and Akt signaling. All these signals are still not well-elucidated in cervical tumors treated by radiation. Also, the underlying mechanism of G0 arrest is worth to be studied, taking into the consideration that $\mathrm{G} 0$ arrest was accompanied by the inhibition of EGFR nuclear translocation. This may suggest an inhibitory effect on non-homologous end-joining DNA-repair (NHEJ), the predominant repair mechanism for DNA-double strand breaks $[28,29]$. Understanding these mechanisms will help in finding more specific targets to the cervical cancer therapy.

In summary, this study provides basic evidence that EGFR could be a potential target to the cervical cancer treatment.

\section{Conflicts of interest}

The authors declared that there is no known conflict of interest.

\section{References}

1. Schiffman M, Castle PE. The promise of global cervical-cancer prevention. N Engl J Med. 2005; 353(20): 2101-2104, doi: 10.1056/NEJMp058171, indexed in Pubmed: 16291978.

2. Ferlay J, Steliarova-Foucher E, Lortet-Tieulent J, et al. Cancer incidence and mortality patterns in Europe: estimates for 40 countries in 2012. Eur J Cancer. 2013; 49(6): 1374-1403, doi: 10.1016/j.ejca.2012.12.027, indexed in Pubmed: 23485231.

3. Zhao Y, Shen L, Chen Xi, et al. High expression of PKM2 as a poor prognosis indicator is associated with radiation resistance in cervical cancer. Histol Histopathol. 2015; 30(11): 13131320, doi: 10.14670/HH-11-627, indexed in Pubmed: 25936600.

4. Kim MK, Kim TJ, Sung CO, et al. High expression of $\mathrm{mTOR}$ is associated with radiation resistance in cervical cancer. J Gynecol Oncol. 2010; 21(3): 181-185, doi: 10.3802/ jgo.2010.21.3.181, indexed in Pubmed: 20922141.

5. Xia S, Zhao Y, Yu S, et al. Activated PI3K/Akt/COX-2 pathway induces resistance to radiation in human cervical cancer HeLa cells. Cancer Biother Radiopharm. 2010; 25(3): 317-323, doi: 10.1089/cbr.2009.0707, indexed in Pubmed: 20578837.

6. Cho S, Cinghu S, Yu JR, et al. Helicase-like transcription factor confers radiation resistance in cervical cancer through enhancing the DNA damage repair capacity. J Cancer Res Clin Oncol. 2011; 137(4): 629-637, doi: 10.1007/s00432-0100925-5, indexed in Pubmed: 20535496.

7. Kim TJ, Lee JW, Song SY, et al. Increased expression of pAKT is associated with radiation resistance in cervical cancer. Br J Cancer. 2006; 94(11): 1678-1682, doi: 10.1038/ sj.bjc.6603180, indexed in Pubmed: 16721365.

8. Chung YM, Kim BG, Park CS, et al. Increased expression of ICAM-3 is associated with radiation resistance in cervical cancer. Int J Cancer. 2005; 117(2): 194-201, doi: 10.1002/ ijc.21180, indexed in Pubmed: 15880373.

9. Qureshi R, Arora H, Biswas S, et al. Mutation analysis of EGFR and its correlation with the HPV in Indian cervical cancer patients. Tumour Biol. 2016; 37(7): 9089-9098, doi: 10.1007/ s13277-016-4789-4, indexed in Pubmed: 26762413.

10. Yarden Y, Sliwkowski MX. Untangling the ErbB signalling network. Nat Rev Mol Cell Biol. 2001; 2(2): 127-137, doi: 10.1038/35052073, indexed in Pubmed: 11252954 .

11. Singh D, Attri BK, Gill RK, et al. Review on EGFR Inhibitors: Critical Updates. Mini Rev Med Chem. 2016; 16(14): 1134-1166, doi: 10.2174/1389557516666160321114917, indexed in Pubmed: 26996617.

12. Li C, Iida M, Dunn EF, et al. Nuclear EGFR contributes to acquired resistance to cetuximab. Oncogene. 2009; 28(43): 3801-3813, doi: 10.1038/onc.2009.234, indexed in Pubmed: 19684613. 
13. Liccardi G, Hartley JA, Hochhauser D. EGFR nuclear translocation modulates DNA repair following cisplatin and ionizing radiation treatment. Cancer Res. 2011; 71(3): 1103-1114, doi: 10.1158/0008-5472.CAN-10-2384, indexed in Pubmed: 21266349.

14. Dittmann K, Mayer C, Rodemann HP. Inhibition of radiation-induced EGFR nuclear import by C225 (Cetuximab) suppresses DNA-PK activity. Radiother Oncol. 2005; 76(2): 157-161, doi: 10.1016/j.radonc.2005.06.022, indexed in Pubmed: 16024112.

15. Marmor MD, Skaria KB, Yarden Y. Signal transduction and oncogenesis by ErbB/HER receptors. Int J Radiat Oncol Biol Phys. 2004; 58(3): 903-913, doi: 10.1016/j.jirobp.2003.06.002, indexed in Pubmed: 14967450.

16. Dittmann K, Mayer C, Fehrenbacher B, et al. Nuclear EGFR shuttling induced by ionizing radiation is regulated by phosphorylation at residue Thr654. FEBS Lett. 2010; 584(18): 3878-3884, doi: 10.1016/j.febslet.2010.08.005, indexed in Pubmed: 20692258.

17. Zhao M, Ma Q, Xu J, et al. Combining CXCL10 gene therapy and radiotherapy improved therapeutic efficacy in cervical cancer HeLa cell xenograft tumor models. Oncol Lett. 2015; 10(2): 768-772, doi: 10.3892/ol.2015.3281, indexed in Pubmed: 26622567.

18. Biscardi JS, Maa MC, Tice DA, et al. c-Src-mediated phosphorylation of the epidermal growth factor receptor on Tyr845 and Tyr1101 is associated with modulation of receptor function. J Biol Chem. 1999; 274(12): 8335-8343, doi: 10.1074/ jbc.274.12.8335, indexed in Pubmed: 10075741.

19. Iida M, Brand TM, Campbell DA, et al. Yes and Lyn play a role in nuclear translocation of the epidermal growth factor receptor. Oncogene. 2013; 32(6): 759-767, doi: 10.1038/ onc.2012.90, indexed in Pubmed: 22430206.

20. Li Z, Hosoi Y, Cai K, et al. Src tyrosine kinase inhibitor PP2 suppresses ERK1/2 activation and epidermal growth factor receptor transactivation by $\mathrm{X}$-irradiation. Biochem Biophys Res Commun. 2006; 341(2): 363-368, doi: 10.1016/j. bbrc.2005.12.193, indexed in Pubmed: 16414009.

21. Santiago A, Eicheler W, Bussink J, et al. Effect of cetuximab and fractionated irradiation on tumour micro-environment. Radiother Oncol. 2010; 97(2): 322-329, doi: 10.1016/j.radonc.2010.07.007, indexed in Pubmed: 20667608.
22. Dittmann K, Mayer C, Wanner G, et al. The radioprotector O-phospho-tyrosine stimulates DNA-repair via epidermal growth factor receptor- and DNA-dependent kinase phosphorylation. Radiother Oncol. 2007; 84(3): 328-334, doi: 10.1016/j.radonc.2007.07.006, indexed in Pubmed: 17714814 .

23. Wanner G, Mayer C, Kehlbach R, et al. Activation of protein kinase Cepsilon stimulates DNA-repair via epidermal growth factor receptor nuclear accumulation. Radiother Oncol. 2008; 86(3): 383-390, doi: 10.1016/j.radonc.2007.10.041, indexed in Pubmed: 18037521.

24. Dittmann K, Mayer C, Fehrenbacher B, et al. Radiation-induced epidermal growth factor receptor nuclear import is linked to activation of DNA-dependent protein kinase. J Biol Chem. 2005; 280(35): 31182-31189, doi: 10.1074/jbc. M506591200, indexed in Pubmed: 16000298.

25. Dittmann K, Mayer C, Rodemann HP, et al. EGFR cooperates with glucose transporter SGLT1 to enable chromatin remodeling in response to ionizing radiation. Radiother Oncol. 2013; 107(2): 247-251, doi: 10.1016/j.radonc.2013.03.016, indexed in Pubmed: 23602371.

26. Jean C, Blanc A, Prade-Houdellier N, et al. Epidermal growth factor receptor/beta-catenin/T-cell factor 4/matrix metalloproteinase 1: a new pathway for regulating keratinocyte invasiveness after UVA irradiation. Cancer Res. 2009; 69(8): 3291-3299, doi: 10.1158/0008-5472.CAN-08-1909, indexed in Pubmed: 19336574.

27. Bozec A, Formento P, Lassalle S, et al. Dual inhibition of EGFR and VEGFR pathways in combination with irradiation: antitumour supra-additive effects on human head and neck cancer xenografts. Br J Cancer. 2007; 97(1): 65-72, doi: 10.1038/sj.bjc.6603791, indexed in Pubmed: 17592499.

28. Rothkamm K, Krüger I, Thompson LH, et al. Pathways of DNA double-strand break repair during the mammalian cell cycle. Mol Cell Biol. 2003; 23(16): 5706-5715, doi: 10.1128/ mcb.23.16.5706-5715.2003, indexed in Pubmed: 12897142.

29. Makita N, Ninomiya I, Tsukada T, et al. Inhibitory effects of valproic acid in DNA double-strand break repair after irradiation in esophageal squamous carcinoma cells. Oncol Rep. 2015; 34(3): 1185-1192, doi: 10.3892/or.2015.4089, indexed in Pubmed: 26135807.

Submitted: 27 November, 2016 Accepted after reviews: 11 May, 2017 Available as AoP: 18 May, 2017 